\title{
Shot Peening Simulation using Discrete and Finite Element Methods
}

\author{
Baskaran Bhuvaraghan $^{\mathrm{a}, *}$, Sivakumar M.Srinivasan ${ }^{\mathrm{b}}$, Bob Maffeo ${ }^{\mathrm{c}}$, Robert \\ D McCLain ${ }^{\mathrm{c}}$, Yogesh Potdar ${ }^{\mathrm{d}}$, Om Prakash ${ }^{\mathrm{e}}$ \\ ${ }^{a}$ GE Aviation, Bangalore, India \\ ${ }^{b}$ Department of Applied Mechanics, IIT Madras, Chennai, India \\ ${ }^{c}$ GE Aviation, Cincinnati, OH, USA \\ ${ }^{d}$ GE Global Research, Niskayuna, NY, USA \\ ${ }^{e}$ ex GE Global Research Research, Bangalore, India
}

\begin{abstract}
Modeling shot peening process is very complex as it involves the interaction of metallic surfaces with a large number of shots of very small diameter. Conventionally such problems are solved using the finite element software (such as ABAQUS) to predict the stresses and strains. However, the number of shots involved and the number of elements required in a real-life components for a $100 \%$ coverage that lasts a considerable duration of peening make such an approach impracticable. Ideally, a method that is suitable for obtaining residual compressive stresses (RCS) and the amount of plastic deformations with the least computational effort seems a dire need.

In this paper, an attempt has been made to address this issue by using the discrete element method (DEM) in combination with the finite element method (FEM) to obtain reasonably accurate predictions of the residual stresses and plastic strains. In the proposed approach, the spatial information of force versus time from the DEM simulation is utilized in the FE Model to solve the shot peening problem as a transient problem. The results show that the RCS distribution obtained closely matches with that of the com-
\end{abstract}

${ }^{*}$ Corresponding author. Address: GE India Technology Center,122, EPIP,Whitefield Road, Bangalore-560066, ph: +919980017207, fax:+918028413113

Email addresses: b. bhaskaran@ge.com (Baskaran Bhuvaraghan), mssiva@iitm.ac.in (Sivakumar M.Srinivasan), bob.maffeo@ge.com (Bob Maffeo), robert.d.mcclain@ge.com (Robert D McCLain), potdar@research.ge.com (Yogesh Potdar), op101.blr@gmail.com (Om Prakash) 
putationally intensive direct FEM simulation. It has also been established, in this paper, that this method works well even in the situations where the robust unit cell approaches are found to be difficult to handle.

Key words: shot peening, finite element method, discrete element method

\section{Introduction}

Shot peening is widely used to enhance the fatigue strength of metals and alloys by inducing residual compressive stress (RCS) on the surface. These stresses also help in reducing failures due to corrosion, stress corrosion cracking, wear etc. Shot peening is used in applications such as peen forming as well. The peening process is shown schematically in Figure.1. It is a complex

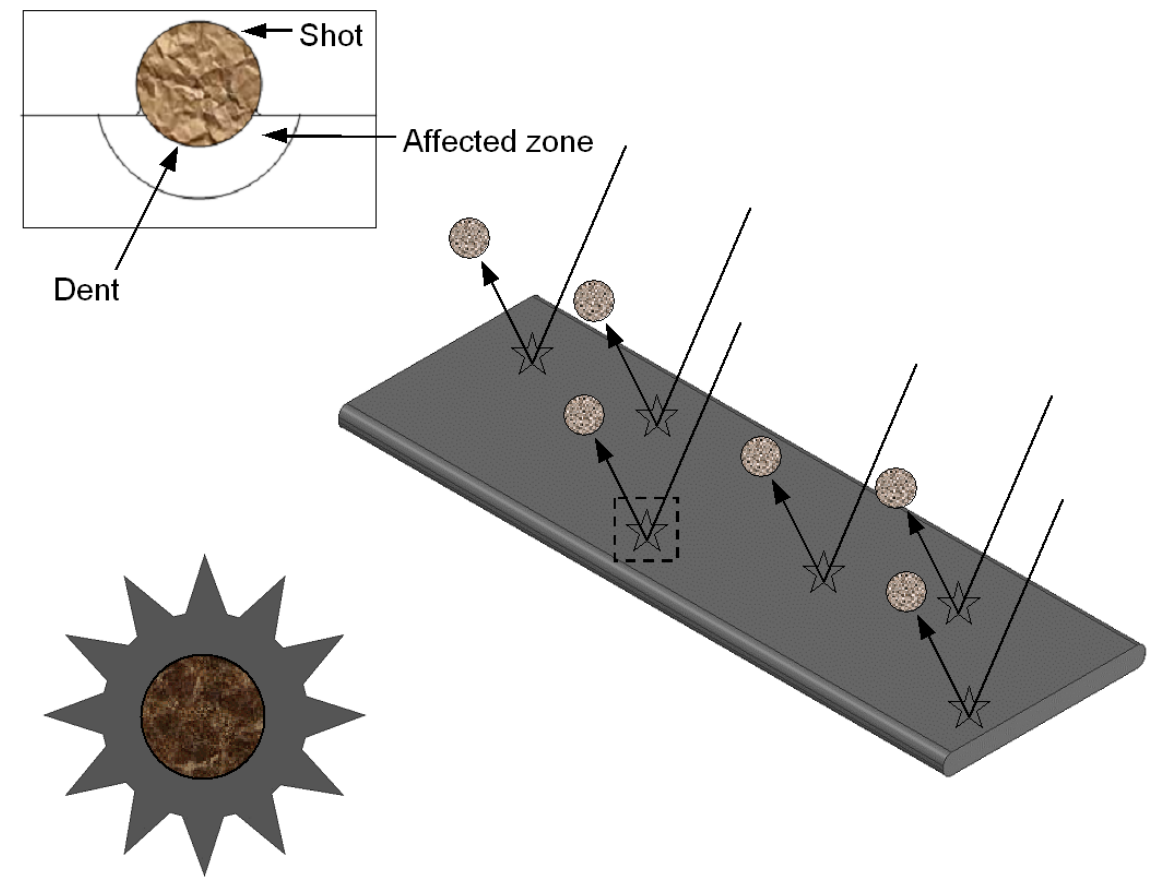

Figure 1: Schematic representation of shot peening

process as it involves millions of shots. Typically, shots are delivered from either a nozzle or a centrifugal wheel. The kinetic energy is derived by the shots either from the pneumatic pressure from the nozzle or the centrifugal force from the wheel. The shot is made in different sizes from different materials such as cast steel, carbon steel, ceramics, glass etc. It is generally 


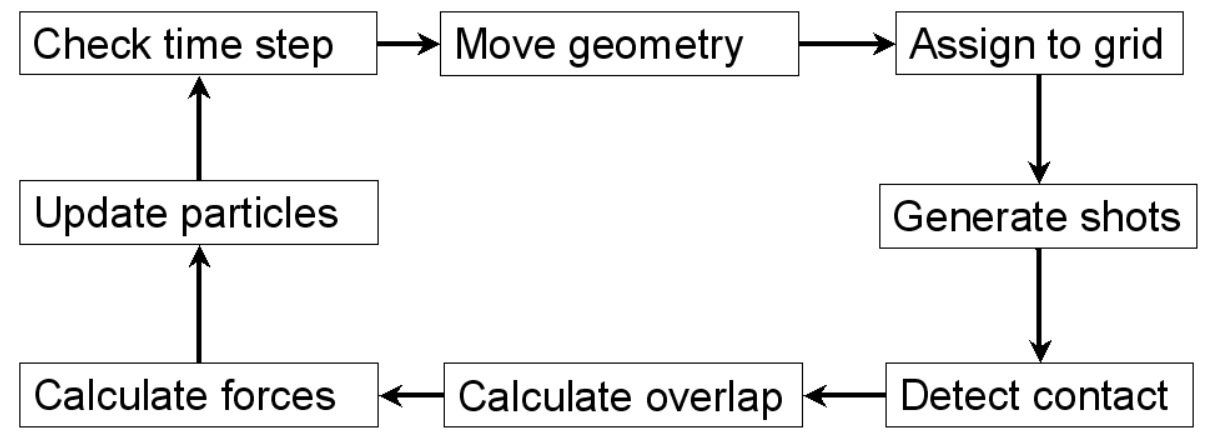

Figure 2: Data flow in DEM. The shots are generated and contacts between the shots and the surface are evaluated. If contact exists, the forces are calculated based on the overlap. The frame is modified to depict post-contact situation. New shots are added and the process continues till the simulation time is completed.

spherical in shape. Shot peening generates cold work and surface roughness along with the RCS.

In order to control the process, many variables related to both shot and target metal surface need to be monitored. The following are the key parameters that affect the work hardening and hence the compressive stresses $[1]$ :

- shot size, velocity, material, hardness

- angle of impact of the shot

- target material and hardness

- friction between shot and target

Understanding the effect of these parameters on the material behavior due to shot peening is important for the designer to reduce the overall cost of the parts.

A large number of studies have been performed on shot peening experimentally. In this approach, parts are shot peened with a set of peening parameters for a certain coverage. Then these parts are subjected to fatigue testing. If the fatigue performance is adequate, then the subsequently manufactured parts are also subjected to the same peening conditions. However, the determination of peening parameters needs to be repeated through further experimentation if the part does not meet the fatigue life, resulting in huge costs. 


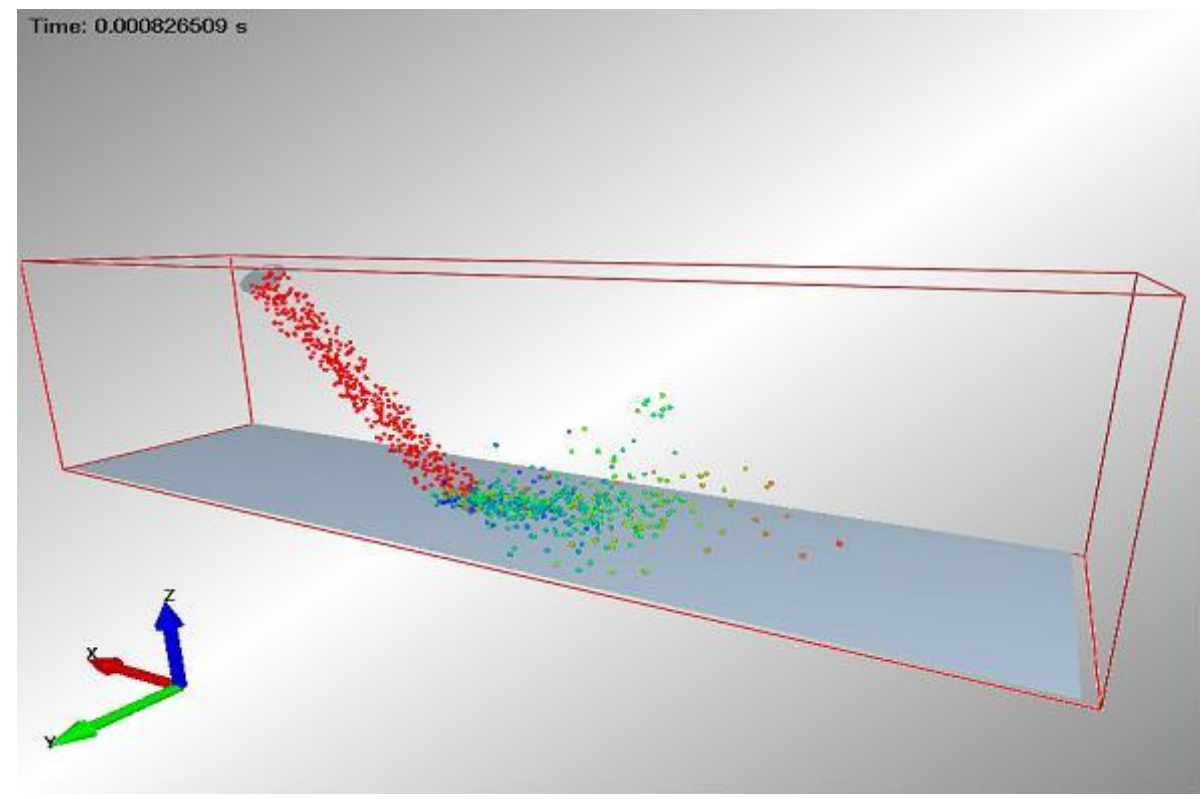

Figure 3: Shot Peening by Baskaran et al (2008). The figure shows the shot peening simulation.

Theoretical methods, therefore, have been employed to predict the RCS to reduce the experimental costs. Details of research using such methods can be found in $[2,3]$. FEM is primarily used in the peening simulation for evaluation of the RCS based on unit cells, as it can accommodate features such as strain rate variations, impact friction and complex material properties. However, there are limitations in using FEM as the numerical tool. In real-life components, the geometry of the features is complex. These features are peened using a large number of shots. The indentation created by each shot is in the order of $0.1-0.3 \mathrm{~mm}$ in diameter. To capture the stress variation, these indentation zones must be further divided into smaller elements. This approach will result in large number of elements for the entire part. A few attempts have been made by the researchers to simulate large number of impacts using FEM. An example in this direction involves impacts of nearly 1000 shots using the explicit-implicit analysis by Wang and Platts[4]. Thus, the complex features of real-life components that are shot peened with a large number of shots are yet to be modeled to capture the residual stresses.

Numerical methods that will handle numerous interactions of particles will be useful to solve this problem. Discrete element method (DEM) is a 


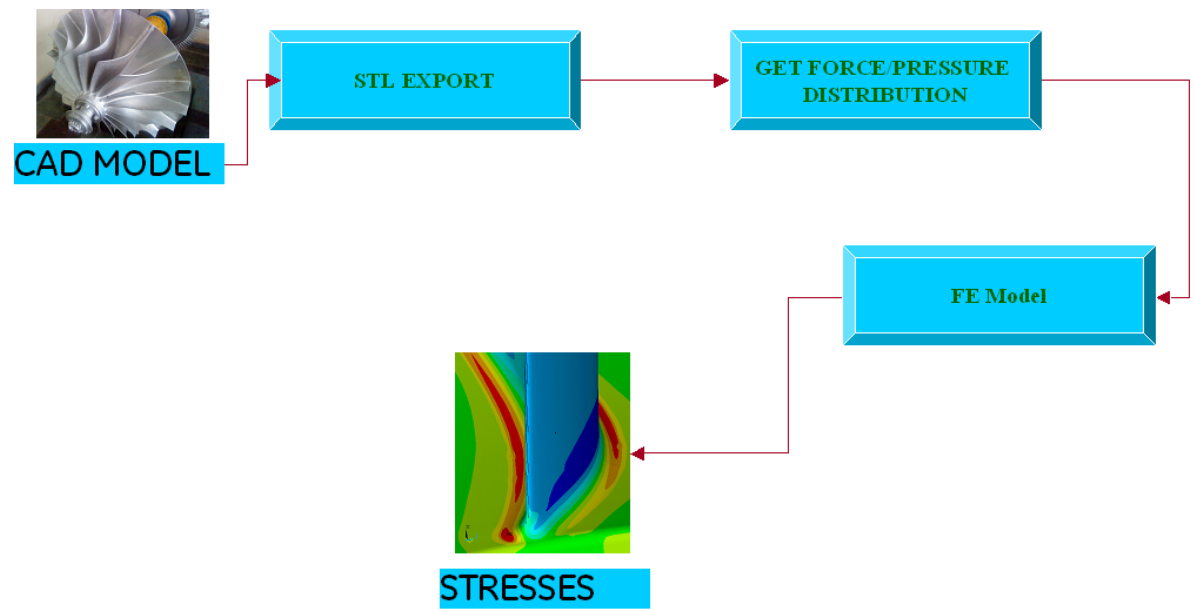

Figure 4: Combined DEM-FEM method indicating the how residual stresses are calculated using DEM and then FEM. The geometry is exported to DEM where the contact force or pressure distribution is obtained. These loadings are applied on FE mesh to get the stresses/strains.

method used in particle simulation and it was originally developed by Cundall et al.[5]. It cannot be directly used in solving a shot peening problem. A typical data flow in DEM is shown in Figure.2. It uses Newton's laws to predict the position, velocity and acceleration of shots. Different contact models exist to predict the contact forces.

An overview of combined DEM-FEM is given in Ref.[6] where the interactions between entities are governed by DEM while each entity is meshed by FEM. Han et al.[7] have performed a two-dimensional analysis treating the sphere as a rigid circle. They have used different interaction laws (linear, Hertz, Winkler and power laws) and also included damping. The work has been extended further to three-dimensional simulation [8]. They have also simulated multiple impacts with a few shots at predetermined locations. The strain distribution due to shot peening is evaluated using combined DEMFEM method at micro-level and the same are applied to the peen-formed component [9]. This method is based on the practical assumption that the stresses and strains on the component surfaces will be the same when they are subjected to the same peening conditions. The big advantage of the method is the use of coarse mesh at the component level. Hong et al.[10] have used DEM to determine the effect of mass flow rate, shot initial velocity and angle of incidence on the energy loss on the target surface. They 
have evaluated the necessary coefficient of restitution (CoR) from shot-shot and shot-plate interactions using ABAQUS Explicit software. Baskaran et al. have used DEM for shot peening simulation and provided a process map to link DEM and FEM [11]. A schematic peening simulation using DEM is given in Figure.3. The method employed to simulate the peening is shown in Figure.4. This process focused on using transient FEM analysis to get the residual stresses from contact pressures, calculated from the contact forces obtained from DEM. This process capability was demonstrated for a single shot impact at an angle of $90^{\circ}$ on the target surface. While the above mentioned researches have used DEM in shot peening simulations, they do not predict the RCS in actual component features employing large number of shots generated randomly. The current procedure employs multiple random shots aimed at calculating RCS more accurately in actual components and their features. It provides comparable results with reduced computational costs. A case study involving peening of a bolt hole is performed using the DEM-FEM method.

The paper is arranged as follows. After a brief overview of the process, the DEM and FEM software tools that are used in the simulation are discussed. Subsequently, different simulations are performed to establish the process for DEM-FEM. In the first simulation, the impact is simulated in FEM as well as DEM to compare the contact parameters from the two different approaches. In the second simulation, the material response in terms of RCS and strains are compared between the methods with impact simulation and contact pressure application in FEM. Enhancements of incorporating infinite elements and CoR due to strain-hardening are considered in the next subsections. The case study of peening the bolt hole using deflector peening is presented in the next section. To perform this simulation, a set of interface programs between DEM with FEM are developed. The techniques that have been employed to reduce the computational resources are also brought out during the discussion on case study. Thus the current process has the ability to combine DEM with FEM to get comparable results with less computing resources.

\section{Sequentially coupled DEM-FEM method}

The current method is shown in the process map (Figure.5). In this study, the process mentioned in [11] is improved to accommodate impacts of multiple shots. 


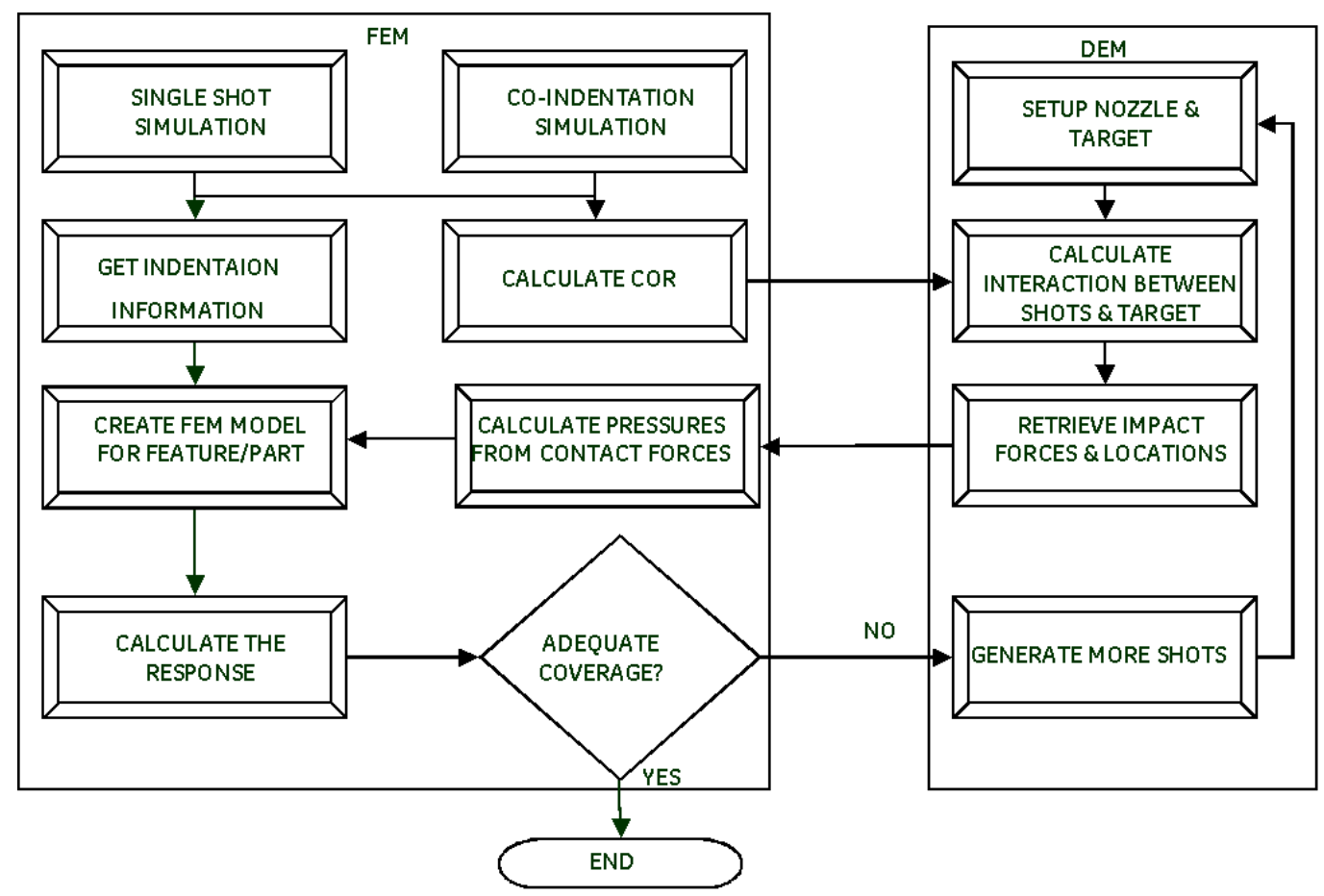

Figure 5: Top level process map. The chart shows the data flow between DEM and FEM simulations.

1. Selection of the appropriate software tools for performing the DEM and FEM simulations (Section. 2.1).

2. A single shot impact analysis is performed in both FEM and a DEMFEM analysis. The responses such as contact duration, force, velocity and momentum are compared. (Section. 2.2). In addition, from the single shot impact simulation, the spatial and temporal pressure distribution along with the respective contact forces are obtained during the contact duration. In addition, the indentation diameter is obtained for defining the pressure zone dimension to be used in the FEM analysis.

3. The effects of applying equivalent pressure in lieu of loads due to single shot impact are evaluated. (Section. 2.3)

4. The infinite element boundaries are used to reduce the the component FE mesh size further (Section. 2.4). The location of such boundaries is optimized for maintaining the accuracy of the RCS prediction.

5. From a multiple co-indentation study, an average CoR is calculated (Section. 2.5). The averaging helps to impart the plasticity effect that 


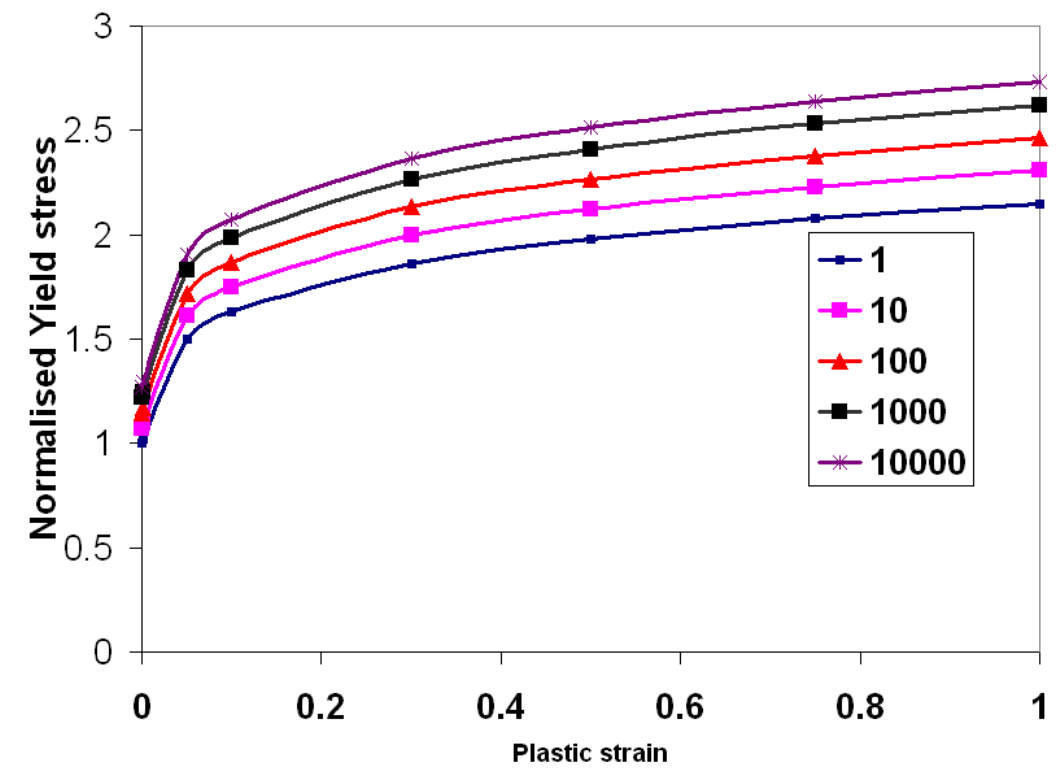

Figure 6: Normalized stress-strain curves for Inco718. The properties are obtained using Johnson-Cook equation.

takes care of strain hardening due to multiple impacts.

6. Once the contact forces and their locations are calculated in DEM, the data is transferred to FEM through a set of programs. Based on the pressure versus contact force look-up table from single shot impact analysis, the contact forces from DEM simulation are converted into equivalent pressure loads. An explicit simulation in FEM is performed using the information obtained from above steps to get the RCS distribution. This is demonstrated through a case study.

The shot is assumed to be rigid and spherical in shape. It is assumed to have the properties of SAE 1070 steel(Table.1). The target material is assumed to be Inco718 whose properties are also listed in the same table. As the impact occurs for a very short duration, strain-rate dependent elastic-plastic properties are more appropriate to use. Figure.6 shows the stress-strain properties of Inco718 for different strain rates. These curves are deduced using appropriate constants Johnson-Cook equation. The yield strengths are normalized with respect to static yield point. 


\begin{tabular}{|c|c|c|c|}
\hline Sl. No. & & Shot & Target \\
\hline 1 & Material & Steel & Inco718 \\
\hline 2 & $\rho\left(\mathrm{kg} / \mathrm{m}^{3}\right)$ & 7800 & 8200 \\
\hline 3 & $\mathrm{E}(\mathrm{GPa})$ & 210 & 211 \\
\hline 4 & $\nu$ & 0.3 & 0.29 \\
\hline
\end{tabular}

Table 1: Properties for shot and target materials

\subsection{Discussions on softwares used}

In the FEM domain, ABAQUS software (version 6.9) from SIMULIA Corporation is used due to its non-linear and impact capabilities [12]. The current study uses EDEM for DEM simulations [13]. The advantages of DEM software are:

- The effects of variation in shot sizes, shapes and velocities can be included.

- Shots can be generated in a random manner with a specified mass flow rate.

- The relative motion of the factory(representing nozzle or wheel) and the target object can be easily modeled.

- Multiple sources of shots from factories can be implemented.

- Orientation and size of the factories can be modeled to be realistic.

- Special peening operations such as hole-peening using deflectors can be easily performed (Section. 3).

The software has the following limiting features that need to be taken care of:

- The collisions are elastic. In shot peening process, the collisions are elastic-plastic. This is circumvented by using the coefficient of restitution(CoR) from FEM analysis.

- DEM does not calculate the stresses and plastic strains automatically. It needs customized post-processing of the results that are inputted into FEM to predict the residual stresses. 
- It provides the information about the location of contact point between shot and the target surface. The forces at the contact point from DEM need to be converted into spatial distribution of pressure over the contact zone in FEM.

- DEM needs coarse mesh while FEM mesh needs to be fine to capture the stress variation.

- The rebound angle is based on the undeformed target surface and the effects of surface variation due to previous impacts are ignored.

\subsection{Comparison of contact parameters between FEM and DEM}

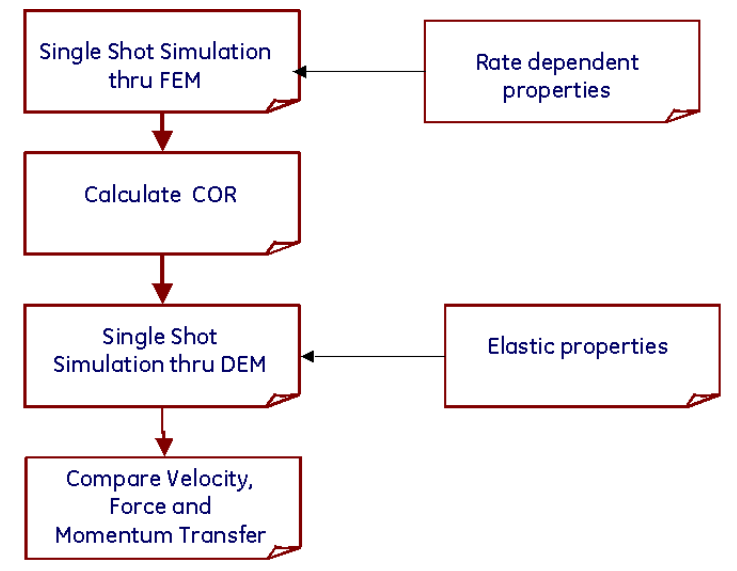

Figure 7: Single shot DEM-FEM Analysis Process

It is assumed that results from the FEM analysis are considered as reference. With a single shot impact analysis in both FEM and DEM methods, it is verified if the force, velocity, contact duration and energy transfer are close. The process map is shown in Figure. 7. The assumptions made in the current approach are:

- The shot is assumed to impact the surface in normal direction.

- The effect of friction is ignored as it does not have much influence on the RCS. 


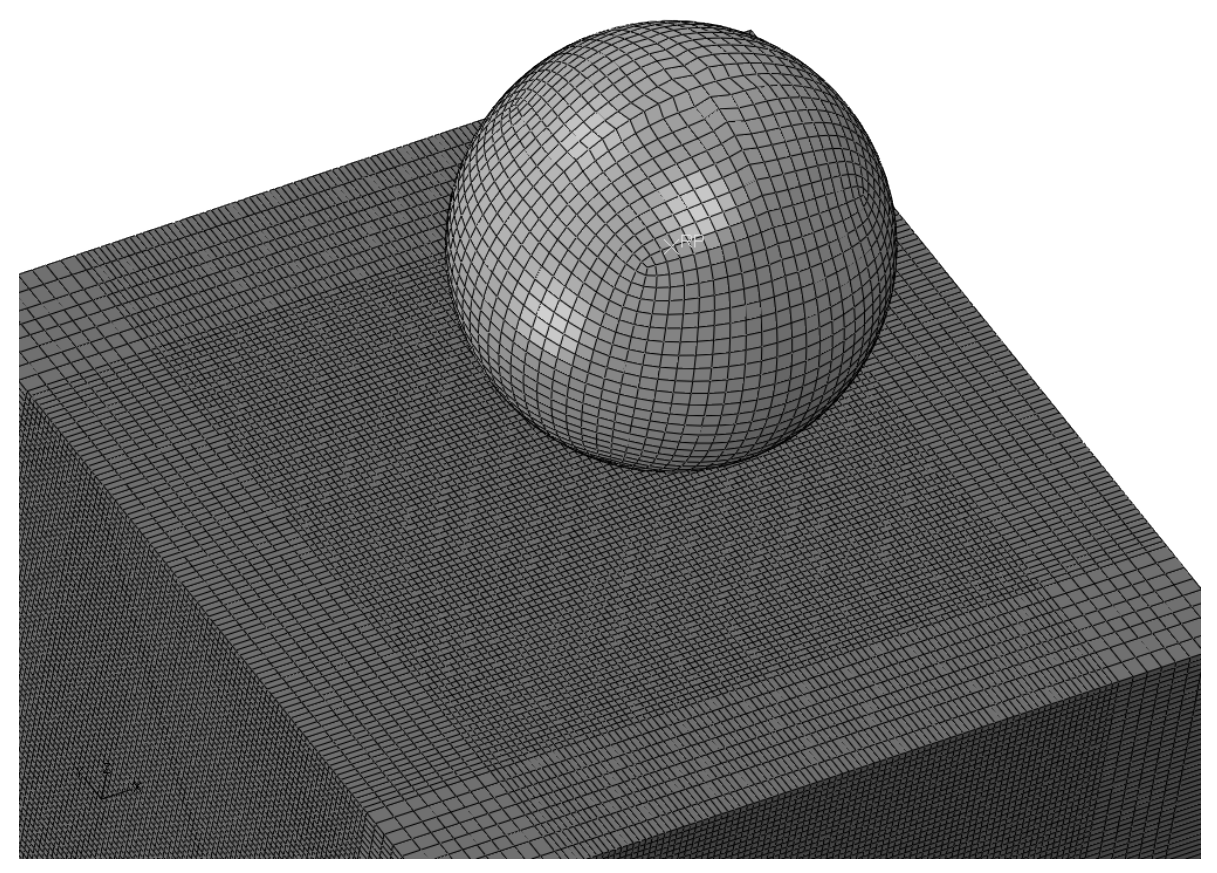

Figure 8: Peening with single shot (FEM)

FEM Method. A unit cell approach is used for single shot simulation. The FE model is shown in Figure.8. The shot is of $0.3556 \mathrm{~mm}$. Symmetry boundary conditions are applied appropriately on the four sides and the bottom surface is vertically supported. Both shot and the target are modeled with C3D8R hexagonal elements with reduced integration. A rigid constraint is created between the center of the shot(reference point) and its elements. The shot properties are given in Table.1. A velocity of $62.5 \mathrm{~m} / \mathrm{s}$ is applied during the impact simulation. Subsequently, a static stabilization run is performed to eliminate the inertial effects.

CoR is calculated from the FEM analysis from the velocities of the shot before and after the impact. The pressure distribution derived from FEM simulation (Figure.9) is almost uniform in the set of nodes within the dent area. The values are normalized with respect to the static yield point, $\left(\sigma_{y}\right)$. As can be seen, the pressure value is about $3 \sigma_{y}$. This result matches with the findings from [14] where plastic impact creates a flat pressure distribution. From this analysis, thus, the following information are captured:

- Coefficient of restitution (CoR) between shot and target surface (to 


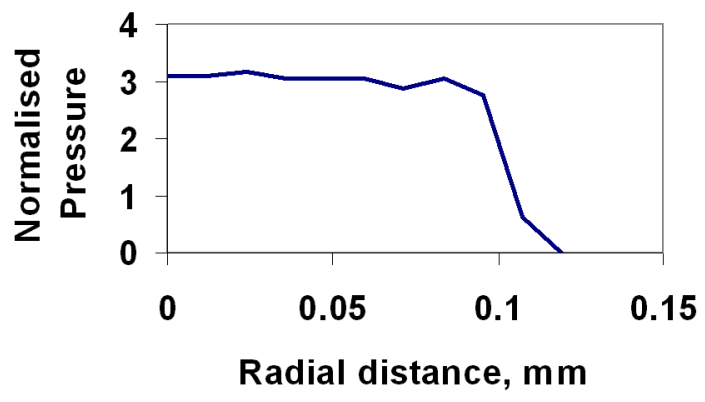

Figure 9: Pressure Application in contact zone. The peak pressure is almost flat.

simulate the energy loss due to plasticity).

- Indentation diameter (for applying the pressure).

- Contact pressure variation along the contact radius with respect to time (to normalize with respect to contact forces from DEM).

DEM Method . In this step, the surface details of the target are captured typically by tessellation through Stereo-lithography (STL) format and have been imported into DEM. Alternatively, a finite element mesh can also be imported into DEM. Typical DEM model is shown in Figure.10, where the sizes of the flat plate and the tiny shots can be compared.

The mesh due to tessellation needs to be at optimum size in order to correctly capture the contact forces. Following are the requirements for DEM:

- The element edge length should be at least as large as the diameter of the shot. A finer mesh is likely to cause the contact overlap spanning across many elements, causing possibly uneven distribution of pressure. Since, the main purpose of the mesh is contact detection, the number of elements must be just adequate to describe the geometry. More elements are likely to increase the simulation time.

- The time step should be not more than $20-40 \%$ of Rayleigh time step which is given by:

$$
T_{R}=\frac{\pi R\left(\frac{\rho}{G}\right)^{0.5}}{0.1631 \nu+0.8766}
$$

where $R, \rho, G$ and $\nu$ are the shot radius, density, shear modulus and poisson's ratio respectively. 


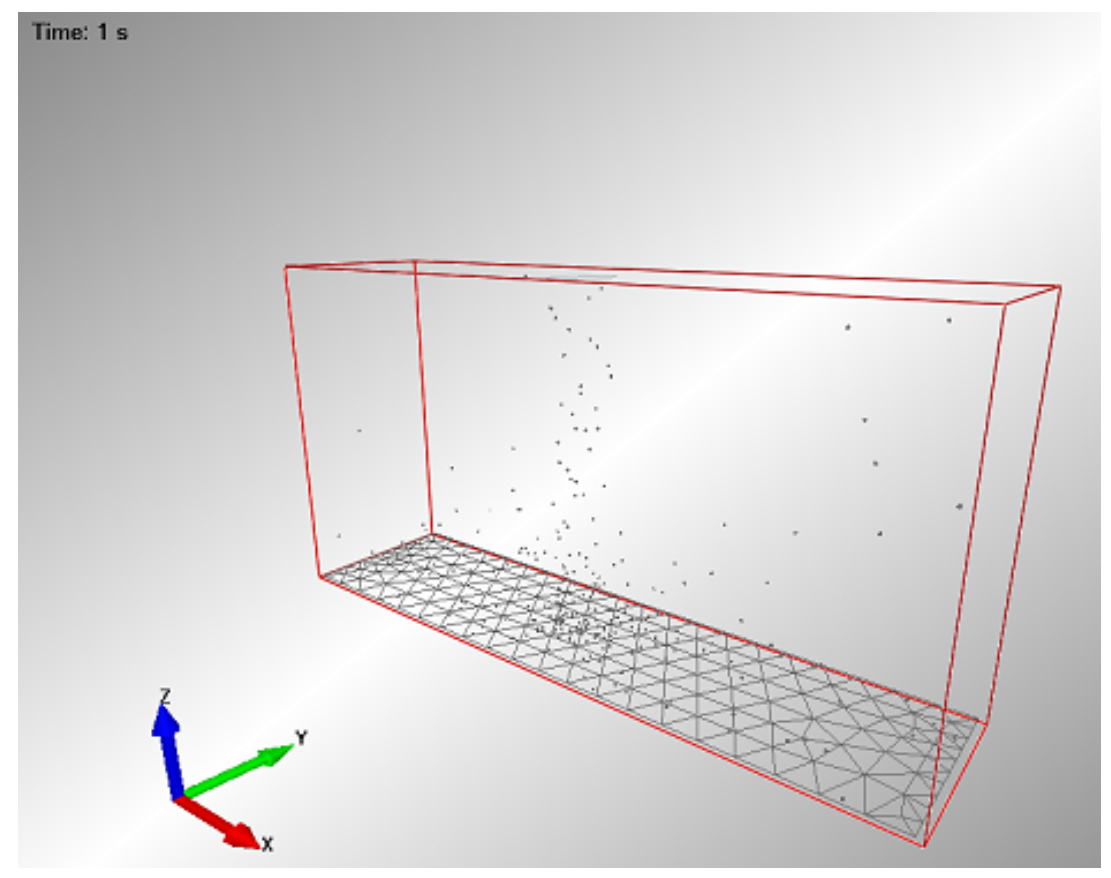

Figure 10: Relative size of triangular mesh and the shots

The same set of shot parameters are applied in the DEM simulation as used in FEM. The CoR of 0.4 is applied from the single shot FEM results. The elastic material properties are input for both shot and the work-piece (Ref. Table.1). The application of CoR simulates the elastic-plastic collision and thus the energy transfer between the shot and the work-piece.

The results are compared in Figure.11 and 12. The velocity after rebound is the same in both cases, as the CoR is kept the same. However, in DEM the drop of velocity and subsequent rebound happens earlier than in FEM. Also, the magnitude of contact force is lower in DEM than in FEM. But the contact duration is the same in both cases. Besides, the total momentum transfered is the same in both cases as compared in the Table.2. Thus based on the values of momentum and contact duration, it can be concluded that the FEM alone and DEM-FEM simulations are very close. The difference in force value can be accounted by normalizing the look-up table properly between contact pressure and contact force. 


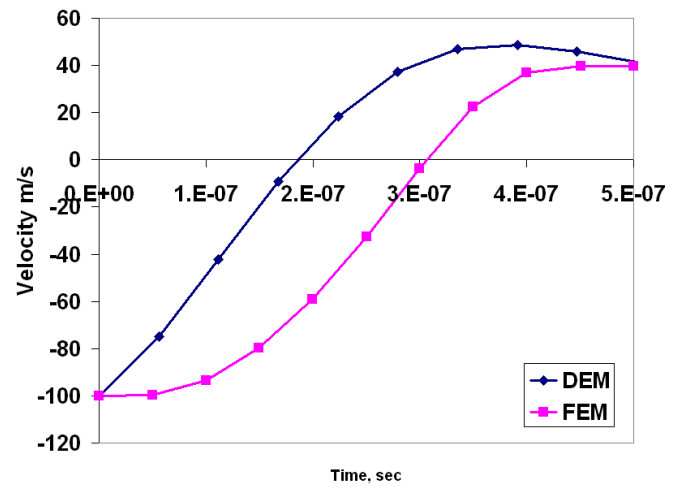

Figure 11: Velocity Comparison between FEM and DEM. The velocity change is quicker in DEM than FEM.

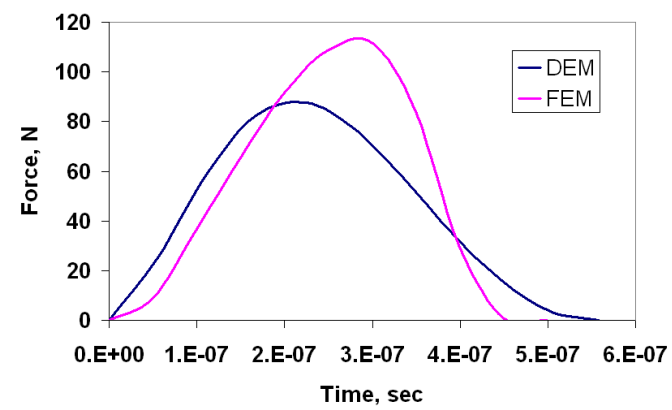

Figure 12: Force Comparison between FEM and DEM. The FEM produces higher contact force than DEM.

\begin{tabular}{|c|c|c|c|c|}
\hline Sl No & Parameter & DEM & FEM & Variation \\
\hline 1 & Maximum Force, N & 87.8 & 111.6 & $27 \%$ \\
\hline 2 & Contact Duration, sec & $5.0 \mathrm{e}-7$ & $5.0 \mathrm{e}-7$ & $0 \%$ \\
\hline 3 & Force x Duration & $2.70 \mathrm{e}-5$ & $2.69 \mathrm{e}-5$ & $0.5 \%$ \\
\hline
\end{tabular}

Table 2: Comparison between DEM and FEM single shot results. The table shows that DEM results can be used to replace contact modeling in FEM, as momentum values are same.

\subsection{Equivalent pressure application}

The next step is to compare the results from a single shot impact on the target material with the results when equivalent pressures are applied. 
Again a FEM based unit cell approach is followed to determine the residual stresses in both cases. The shot is assumed to impact the surface in normal direction. The pressure distribution is shown in Figure.9. Figure.13 shows the normalized stress with respect to static yield point of Inco718 for both cases. The stresses due to shot impact and equivalent pressure application match very well indicating that the process can be used for the features of actual parts. Since the contact detection is totally eliminated, the simulation is faster when contact pressures are applied as can be seen in Table.3.

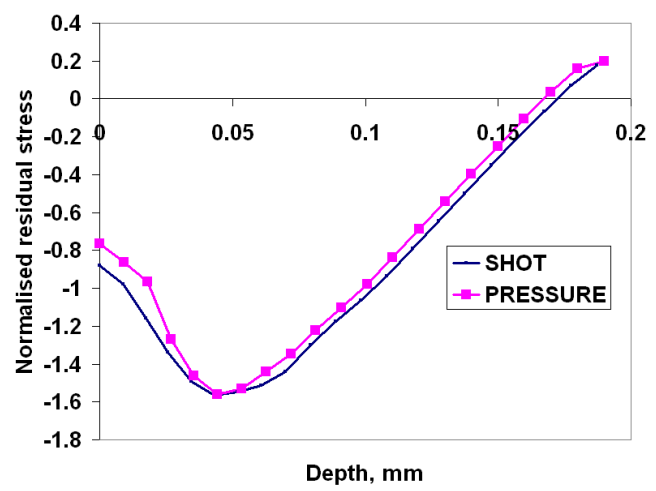

Figure 13: Stress due to impact versus pressure application. This investigation has been done to apply equivalent pressures in stead of forces.

\begin{tabular}{|c|c|c|}
\hline Sl No & Loading Type & CPU Time \\
\hline 1 & Single Shot Impact & $19.1 \mathrm{sec}$ \\
\hline 2 & Pressure Application & $14.1 \mathrm{sec}$ \\
\hline
\end{tabular}

Table 3: Comparison between single shot and pressure application results. Pressure application reduces the simulation time as no contact detection is involved.

\subsection{Using infinite elements}

The usage of DEM practically eliminates the need of modeling shots in FEM in our current approach. Only the target component needs to be idealized in FEM. As the surface stresses are of main interest, the mesh has to be very fine in the surface layers. Therefore, even without the need to model the shots, the current approach can lead to enormous number of elements. 
As shot peening is a surface phenomenon, if the meshing of inner material can be avoided, it will reduce the FEM model size. The ABAQUS software has the feature of infinite element that absorbs the shock wave propagation. This feature is utilized to avoid meshing of inner material by coating the finite elements with a layer of infinite elements. For example, a 3D analysis on unit cell has been performed by Schwarzer for simulating multiple impacts using ABAQUS-explicit tool that uses infinite boundaries used to dampen the stress oscillations[15]. Thus, a layer of infinite elements can be included at the appropriate location beyond the finite elements in the current process to avoid the meshing of the inner material of the part as shown in Figure.14.

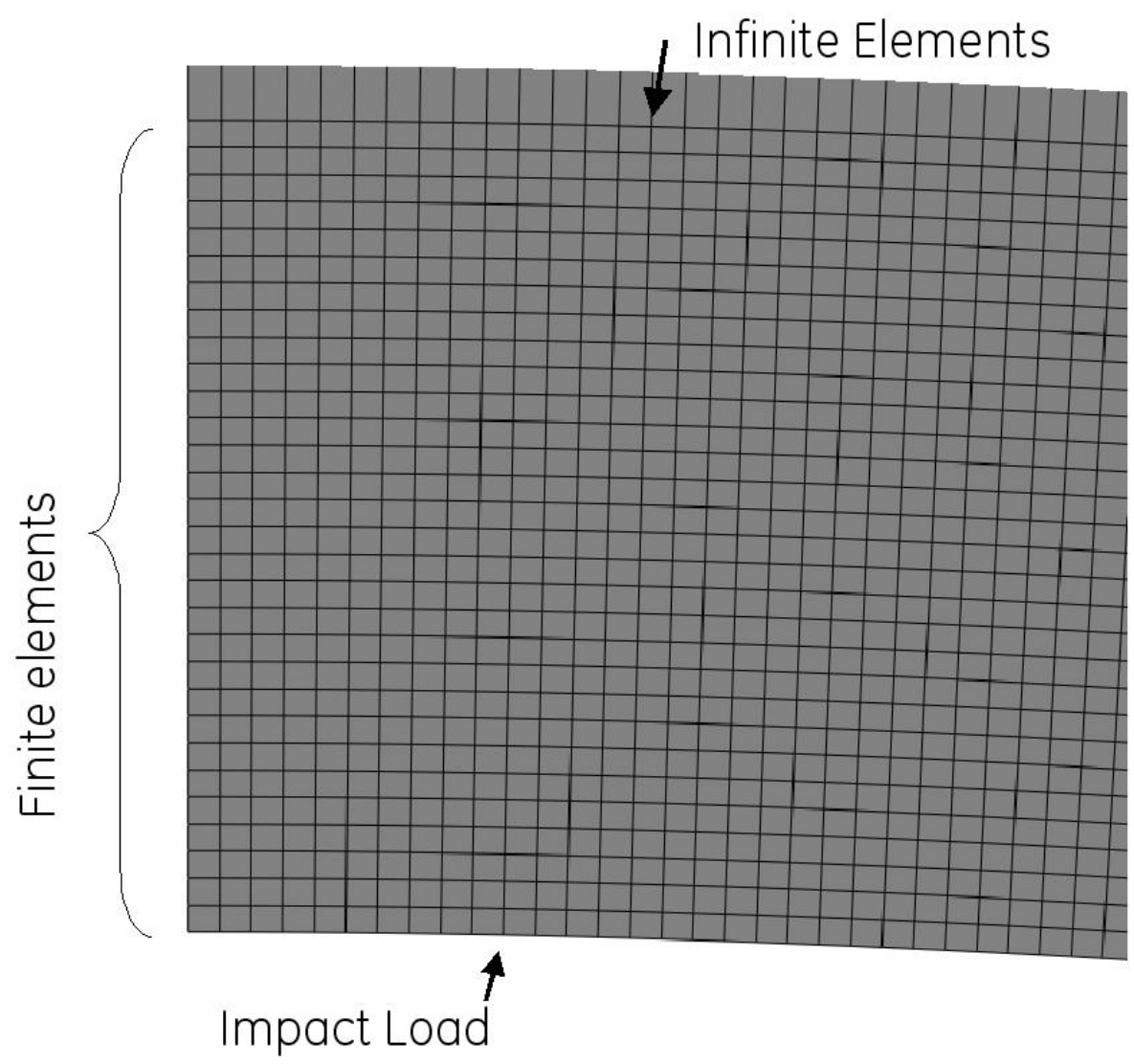

Figure 14: Modeling of infinite elements. A layer of infinite elements is added at the outermost FEM layer normal to the wave direction. 
A set of runs using FEM have been made to determine the minimum depth to which the finite elements need to be present. The target material is assumed to be Inco718, whose properties are given in Figure.6. The FEMonly mesh is supported at the bottom surface in the direction of the impact. The FEM-infinite element mesh has no support in the vertical direction. The same set of shot and target parameters, which have been used in the previous analyses, are used. For the given set of shot properties and target material, it is found that a minimum depth of $0.375 \mathrm{~mm}$ is required for the finite element mesh (Figure.14) to develop the same RCS distribution when it is supported at the bottom. If the FE mesh is thinner than this, sufficient and correct distribution of RCS is not developed when a layer of infinite elements is present. Thus, the layer of material provides enough resistance for RCS to develop, when the thickness of FE mesh is significant. This is a function of shot parameters (such as size, velocity, angle) and the target material properties (such as hardness). Figure.15 compares the stresses, normalized with static yield point, for the cases with and without infinite elements developed in a thickness $0.375 \mathrm{~mm}$.

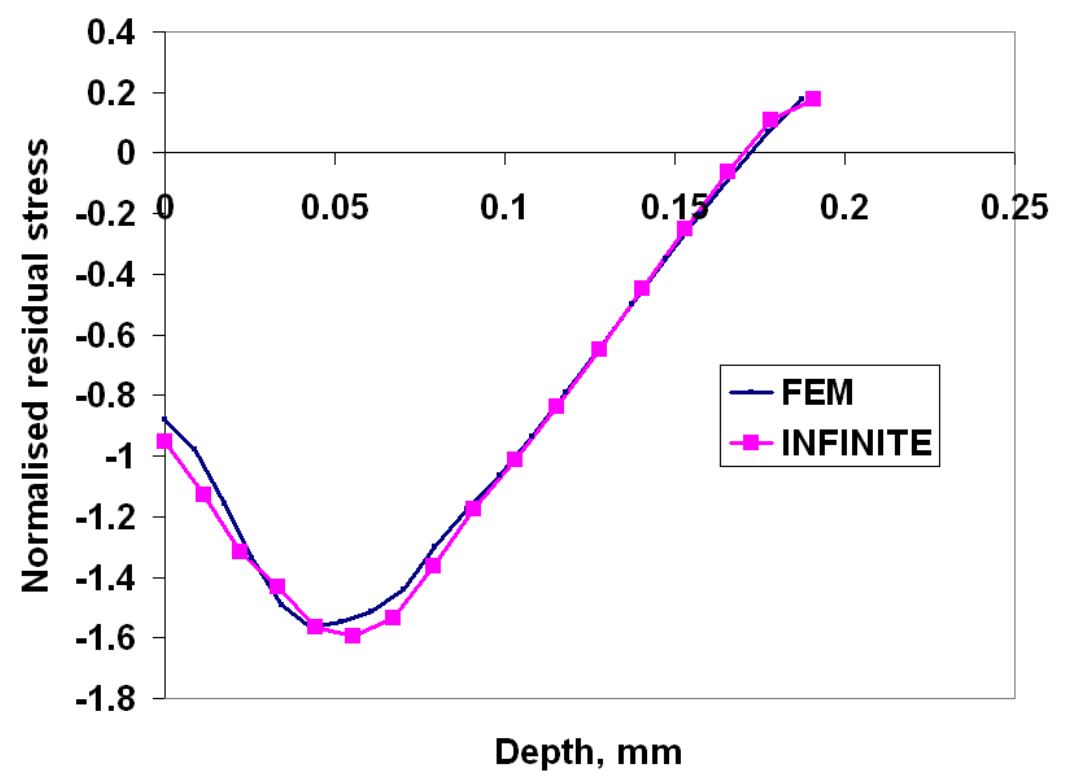

Figure 15: Comparison of stresses with and without infinite elements. RCS in both cases are close enough to use infinite elements in stead of modeling the entire inner material. 


\subsection{Calculation of $C o R$}

The CoR is a function of shot velocity, shot material, angle of impact, target material hardness etc. For a given set of shot, target material, velocity and nozzle orientation, the CoR as calculated from FEM software is assumed to be a constant. The CoR will be higher due to the higher dynamic yield strength of the target material at higher strain rates. To have a $100 \%$ coverage, when the shots are delivered in a random basis it is required to have 3-4 times the number of shots that will be required when they are positioned in an orderly manner. In other words, each point is likely to be hit 3-4 times in an average sense [16]. It implies that as the target material is strain-hardening, subsequent impacts at the same location is likely to hit a harder surface than the previous ones. To take into the effect of strain hardening in the calculations, a FEM analysis with multiple impacts at the same location has been performed and the average CoR has been evaluated. The Table. 4 shows the variation of CoR due to strain-hardening effect of the

\begin{tabular}{|c|c|c|c|}
\hline Impact No & $\begin{array}{c}\text { Input velocity } \\
\mathrm{m} / \mathrm{sec}\end{array}$ & $\begin{array}{c}\text { Rebound Velocity } \\
\mathrm{m} / \mathrm{sec}\end{array}$ & $\mathrm{CoR}$ \\
\hline 1 & 100 & 40.27 & 0.396 \\
\hline 2 & 100 & 54.04 & 0.540 \\
\hline 3 & 100 & 58.28 & 0.583 \\
\hline 4 & 100 & 76.31 & 0.763 \\
\hline
\end{tabular}

Table 4: Variation of CoR due to strain-hardening. Due to successive impacts, the material hardens further resulting in increased CoR.

target material. The average CoR is found to be 0.57 .

\section{Case study: Deflector peening of a bolt hole}

The next step is to simulate multi-ball simulation in DEM. The test case chosen is peening simulation of a bolt hole. A special method called deflector peening is employed to peen the holes which are small in size. The bolt hole in consideration is $7.5 \mathrm{~mm}$ in diameter and $25 \mathrm{~mm}$ long. A rigid rod is used to deflect the shots from the nozzle onto the hole surface (Figure.16).

The process can be divided into four steps. In the first step, impact analysis is performed on a unit cell with single shot. This step enables us to capture the required information (such as indentation diameter, pressure values for respective contact force magnitudes over the contact duration) 


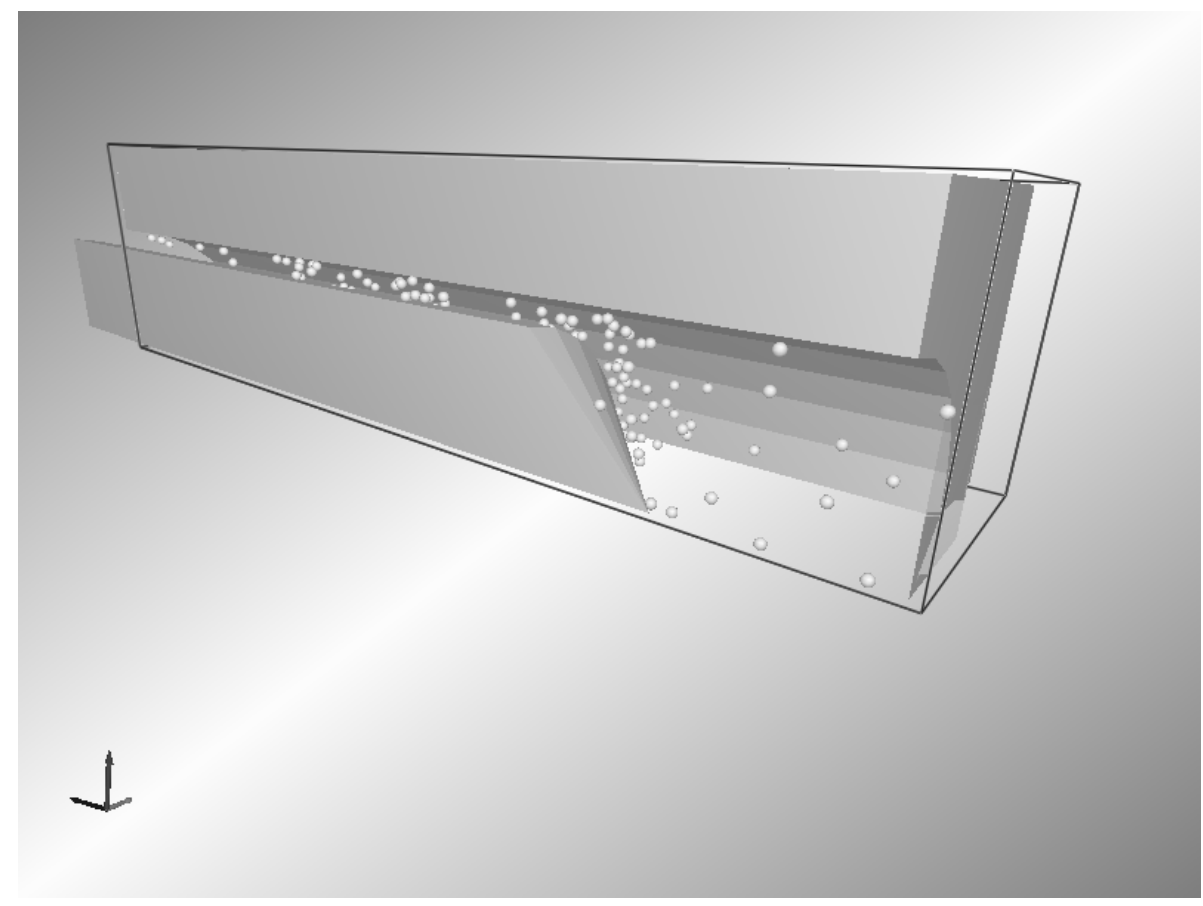

Figure 16: Deflector peening of bolt hole. The shots from nozzle are deflected by the rod onto the bole hole.

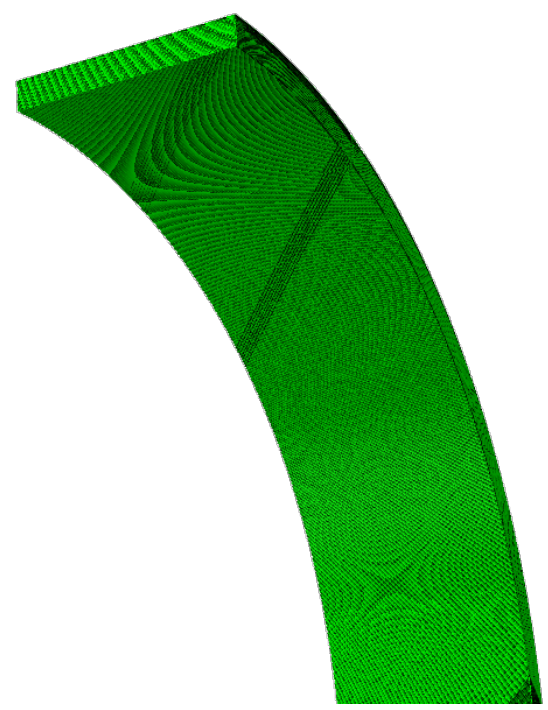

Figure 17: Bolt hole mesh. The model uses reduced integration elements in the impact zone and one layer of infinite elements. 
for the next step which is a DEM analysis. In the third step, the results from DEM native format are translated to FEM-specific format. Again we perform FEM analysis in the fourth step using the results obtained from DEM analysis.

Step 1-Single shot FEM analysis:. A unit cell is used for the FEM simulation. The shot and target material are the same as before. The shot diameter is also $0.3556 \mathrm{~mm}$. The FE model is shown in Figure.8. From this analysis, thus, the following information are captured:

- Coefficient of Restitution (CoR) between shot and target surface (to simulate the energy loss due to plasticity).

- Indentation diameter (for applying the pressure).

- Contact pressure variation along the contact radius with respect to time (to normalize with respect to contact forces from DEM).

Then, a look-up table between the contact force and maximum pressure from single shot analysis is established.

Step 2-DEM analysis:. The surface of the CAD model is imported in DEM software. The CoR between shot and target surface is assumed to be 0.57 as calculated before in multiple shot simulation. The factory that represents the nozzle is designed to generate the shots at any angle to the target plate with a specified velocity. The mass flow rate and the duration of simulation is adjusted to be in line with the actual process. The gravity effects can also be added if required. The rate of delivery of shots is assumed to be around $40000 / \mathrm{sec}$ which is equivalent to the mass flow rate of shots. The deflector rod is given a translational velocity so that it moves from one end of the hole to the other along the axis of the hole. The shots from the nozzle impact the rod and then deflected on the hole surface. There can be impacts between shots as well. The shots that leave the domain are ignored for further analysis as discussed before. The FE mesh of a part of the hole is shown in Figure.17.

The DEM program calculates the forces due to the shot impacts and the location of such collisions. It provides the following results during the simulation:

- Contact point coordinates

- Contact force component magnitudes 
- Friction force component magnitudes

Since, friction does not play a big role in the magnitude of residual stresses, the effect of friction is ignored. The remaining data are used to apply the pressure loading on the FEM model.

Step 3-Transfer of data from DEM to FEM. These forces are transferred in suitable format to the FEM software using a set of translator program. A PERL language based script has been developed to transfer these data from the native DEM format to FEM loads format. The process of capturing these data into FEM format is depicted in the flow chart (Figure.18). The PERL script needs:

- the surface nodes and the connectivity of the elements on the surface from FE model.

- the contact point coordinates and the components of contact forces from the results obtained from DEM.

First the set of FE nodes that form the target surface are sorted in $\mathrm{X}, \mathrm{Y}$ and then $\mathrm{Z}$ directions. The sorting is done to speed-up the selection of node from FEM model for every contact point determined through DEM analysis. Next the contact locations and the forces are extracted from the DEM output. From the coordinates of every contact point from DEM simulation, the nearest node on the FEM model is determined. Based on the indentation radius from single shot FEM results, the adjoining nodes and connected elements in the bolt hole FE model are selected. These zones define the pressure application areas. From the contact force calculated from DEM analysis, the pressure distribution is interpolated. To perform the interpolation, first a force factor is determined as the ratio of maximum force from FEM to the maximum force from DEM. From the look-up table calculated from single shot impact analysis, the pressure distribution for every impact is determined based on the force factor.

During the DEM simulation, the output from DEM is generated at fixed time intervals. In DEM results, the forces due to single impact at a contact location can spread over multiple time intervals. The program captures such impacts and combine them to a single impact by extracting the contact location and maximum force components for each impact. If the impact occurs on an edge or corner of the tessellation in DEM, the program has the ability to eliminate redundant data and it retains only one set of data. 


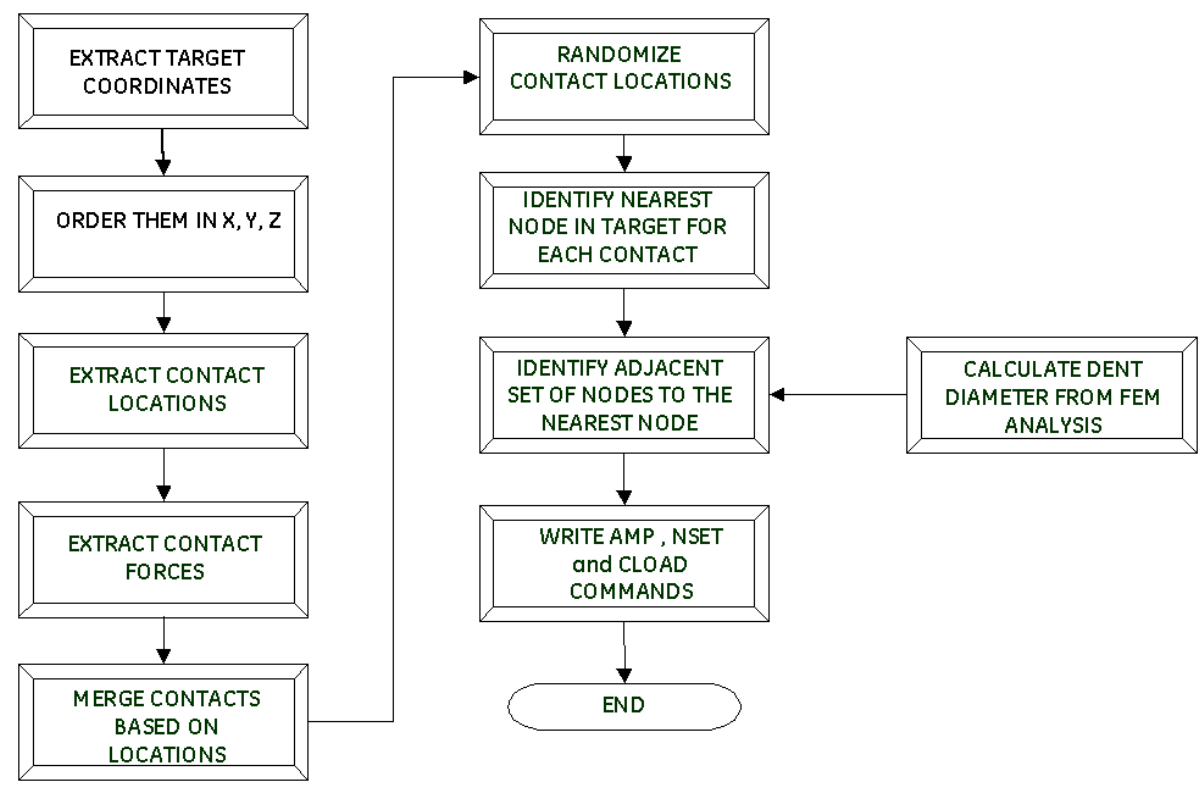

Figure 18: Data extraction process from DEM to FEM. The contact forces and locations are extracted from DEM and converted to equivalent pressures for FEM analysis.

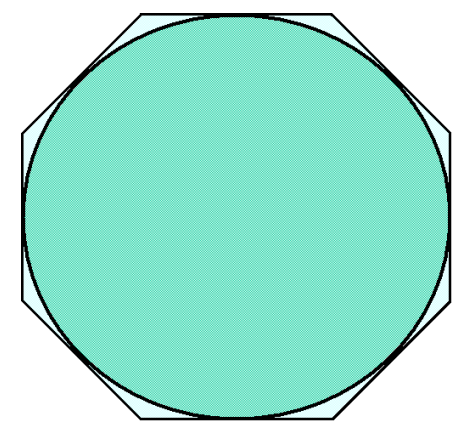

Figure 19: Loading zone identification. The circular pressure zone is modeled as a octagonal zone to follow the mesh pattern.

Step 4- FEM Analysis. The work-piece is modeled in FE software with fine mesh that can capture the pressure variations as the contact occurs. The surfaces on the sides of the hole mesh are applied with symmetry boundary conditions appropriately. The pressure zone is assumed to be octagonal. As the meshing is performed with 8-noded C3D8R brick elements, the size of the octagonal zone is chosen to be close to a circular pressure zone(Figure.19). The analysis is done as a transient explicit dynamic analysis to predict the 


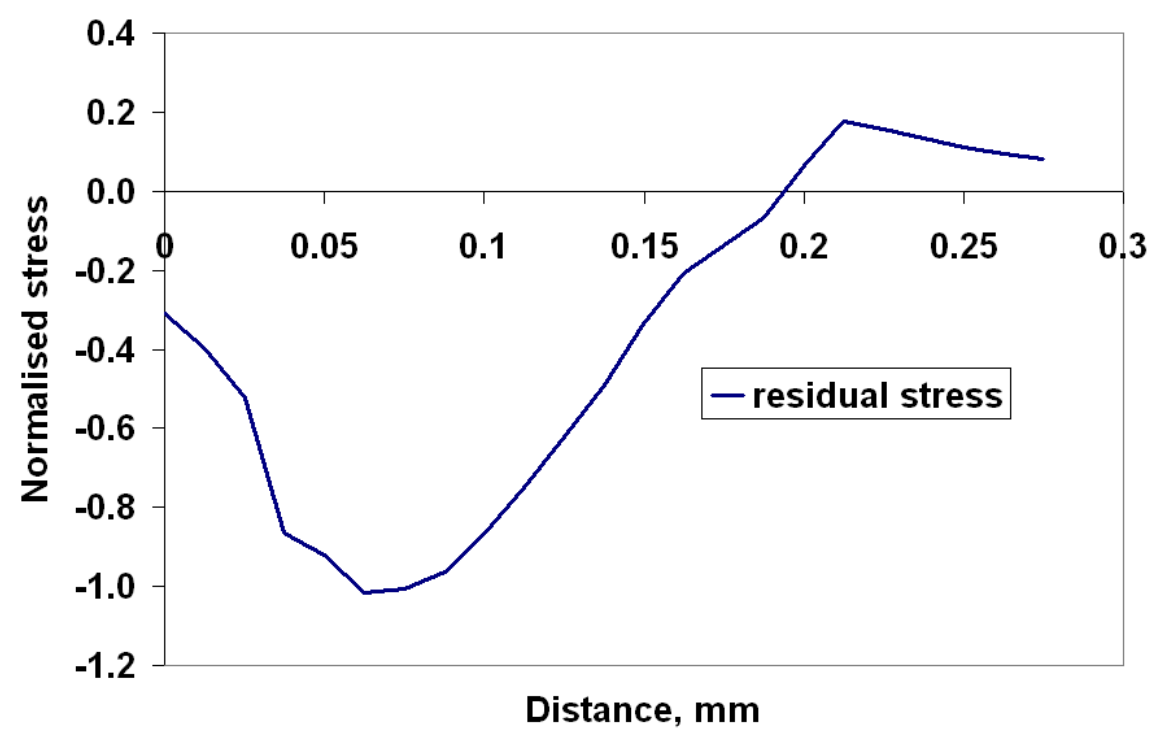

Figure 20: RCS distribution during multi-shot simulation. It can be observed that the RCS is very similar to the observed phenomenon.

stresses accurately so that inertial effects are also captured.

\section{Results and Discussions}

\subsection{Residual compressive stress}

Multiple impact simulation is performed on the bolt hole. Figure.20 shows RCS distribution. These values are captured in the location where a single shot impact is present. Due to the orientation and velocity component, a load factor of 0.7422 is used at this location. Figure.21 depicts the impact locations of the shots during the simulation. As can be seen in the figure, the first round of impact simulation of bolt hole peening has a very low coverage. To get $100 \%$ coverage, the DEM simulations have to be repeated more times, but FEM simulation has to be performed only once.

\subsection{Reduction of computing resources}

Different measures that have been implemented have resulted in significant reduction of memory and hard disk requirements and improving the simulation time. 


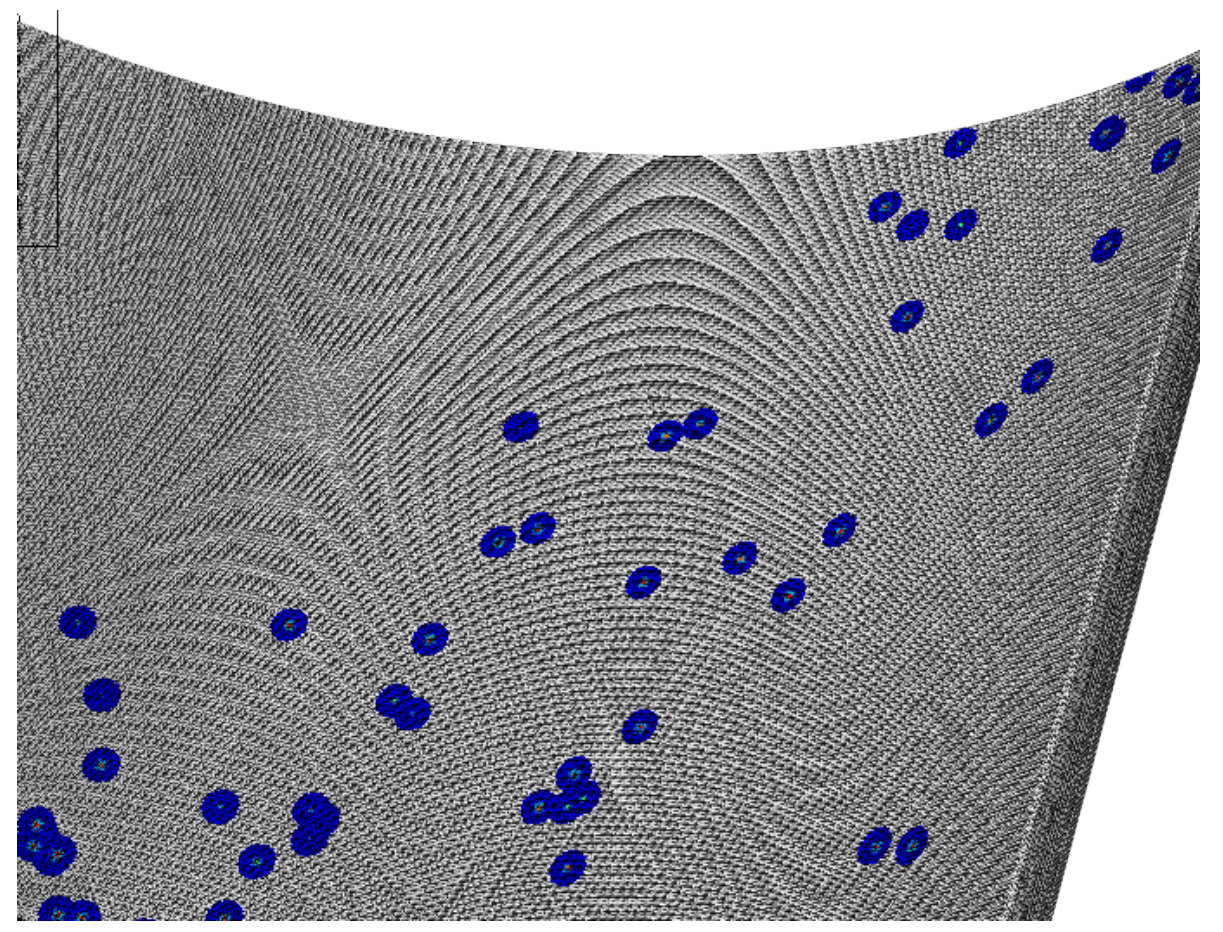

Figure 21: Impact locations on bolt hole. These locations are generated during DEM simulation and transferred to FEM simulation through the translator programs.

FEM model size. The DEM-FEM approach has eliminated the need to model the shots in FEM, thus reducing the model size many folds. Still, the number of elements for the target in FEM required to predict the stresses accurately remains very high as we want to capture the RCS variation due to contact pressure during impact. This necessitates very high computer memory and disk space. Including the infinite elements appropriately has helped to avoid the modeling of inner material without sacrificing any accuracy.

FEM simulation time. The simulation time in FEM has been significantly reduced by avoiding contact detection. Further speeding up of FEM runs has been accomplished by grouping shots that are far away from each other. If the RCS fields of far-away shots are not influenced by each other, then those impacts are assumed to start at the same time. Figure.22 shows that two shots impacting simultaneously have no bearing on each other's stress region when the distance between them is equal to or more than 1.5 times the shot diameter. Using this result, the impact locations of shots that are far away 
from each other are scanned and grouped. The translator program groups such impacts to the same start-time to reduce the number of Amplitude curves that are generated for ABAQUS/Explicit run.

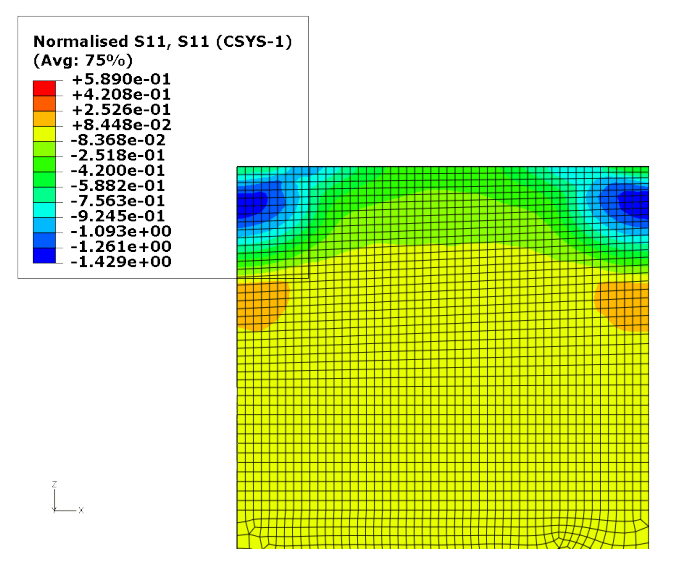

Figure 22: Effect of simultaneous impacts of two shots. Beyond 1.5 times shot diameter, the stress fields from the two impacts have very less effect on each other.

Simulation time in DEM. The duration has been reduced by suitably adding more nozzles that do not interfere with each other through their shot delivery can significantly speed up the process. In a different example, the authors have used two nozzles simultaneously to peen the root fillet of a blade airfoil (Figure.23). One nozzle has been used for leading edge and the other for trailing edge.

Computing resources in DEM. The simulation of shot peening process using DEM software demands large computer resources in terms of disk space. It can exceed the file size limits for simulations involving several time steps. This limitation has been overcome by splitting the simulation process into multiple steps and finally merging the results files.

\section{Conclusions}

The contact forces due to random peening obtained from DEM are applied on the FEM model to make the residual stresses to be more realistic. The necessary computing resources are reduced by efficient contact detection in DEM (that eliminates the same in FEM) and elimination of shots outside 


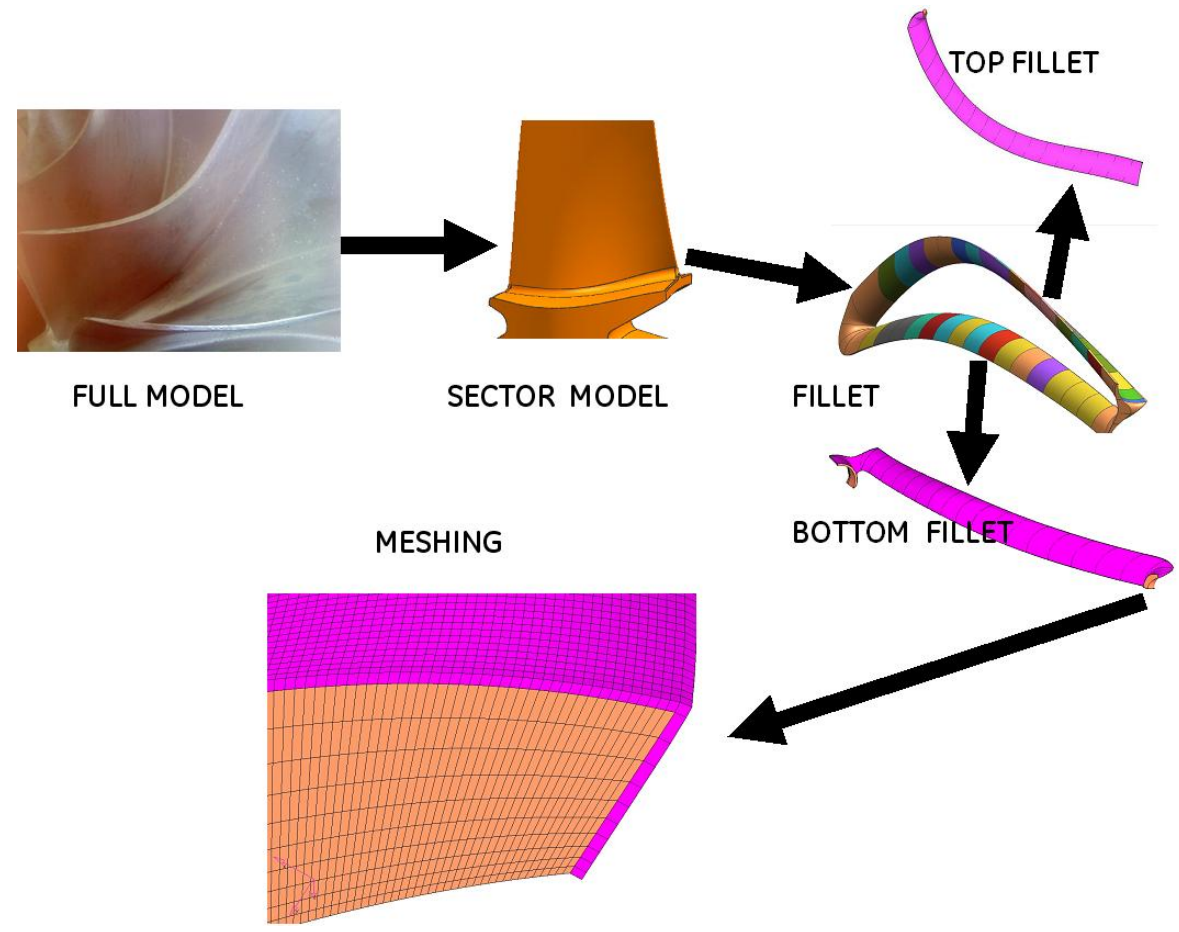

Figure 23: Speeding the simulation for fillet region. Two nozzles, one for pressure face and another for suction face, are employed to reduce the simulation time.

the domain in DEM. Besides, splitting the DEM runs helps to manage disk space efficiently and adding multiple nozzles in DEM to speed-up the peening. Significant saving also has been achieved by modeling of infinite elements in FEM to reduce the model size and effective combining of start time of faraway shots. At the same time, the accuracy of results by using appropriate material models in FEM is maintained, by using strain-rate dependent target properties and corresponding CoR.

Thus, combining DEM results and FEM provides an efficient tool to model the real-life feature of components such as bolt holes. Thus, this process paves way for shot peening simulations on large components to get realistic stresses.

The next ongoing steps are to get $100 \%$ coverage and to superpose the predicted values of RCS and cold work on the surface of the components. The seminal work done by Han et al.[9] can be used to superpose the stresses and strains. The superposition step is important so as to subject the part 
with RCS under service loads such as aircraft engine mission. In such cases, the material model needs to be revalidated to cover the relaxation behavior of the material.

Acknowledgments. We like to thank GE Aviation for supporting the work and EDEM solutions for technical discussions. We also like to acknowledge the help provided by our colleagues Sai Krishna and Mrinal Shukla.

\section{References}

[1] A. Niku-Lari, Shot peening, in: ICSP-1, Paris, 1981, pp. 1-21.

[2] B. Baskaran, S. M. Sivakumar, O. Prakash, Y. Potdar, B. Maffeo, P. Domas, Overview of the effetcs of surface enhancement processes on plastic strain, work hardening and residual stresses, in: ICSP-10, Vol. 2008049, Tokyo, Japan, 2008, pp. 81-86.

[3] R. C. McClung, A literature survey on the stability and significance of residual stresses during fatigue, Fatigue and Fracture of Engineering Materials and Structures 30 (3) (2007) 173-205.

[4] T. Wang, J. Platts, Finite element impact modelling for shot peen forming, in: ICSP-8, Garmisch-Partenkirchen, Germany, 2002, pp. 540-546.

[5] P. Cundall, O. Strack, A discrete numerical model for granular assemblies, Geotechnique 29 (1979) 47-65.

[6] A. Munjiza, The Combined Finite-Discrete Element Method, Wiley, 2004.

[7] K. Han, D. Peric, A. Crook, D. Owen, A combined finite/discrete element simulation of shot peening processes part I- study of 2D interaction laws, Engineering Computations 17 (5) (2000) 593-619.

[8] K. Han, D. Peric, D. Owen, J. Yu, A combined finite/discrete element simulation of shot peening processes part II-3D interaction laws, Engineering Computations 17 (6-7) (2000) 680-702.

[9] K. Han, D. Owen, D. Peric, Combined Finite/Discrete element and Explicit/Implicit simulations of peen forming processes, Engineering Computations 19 (1-2) (2002) 92-118. 
[10] T. Hong, J. Ooi, J. Favier, B. Shaw, A numerical simulation to relate the shot peening process parameters to the induced residual stresses, in: ICSP-9, Paris, France, 2005, pp. 100-106.

[11] B. Baskaran, S. M. Sivakumar, Y. Potdar, D. Paul, A. Srikanth, P. Weitzman, Shot peening modeling by combining DEM and FEM, in: ICSP-10, Vol. 2008049, Tokyo, Japan, 2008, pp. 131-142.

[12] ABAQUS User Manual Version 6.9, Hibbitt, Karlsson \& Sorenson, Inc, 2009.

[13] EDEM 1.3 User Guide, DEM Solutions, 2007.

[14] K. Johnson, Contact Mechanics, Cambridge University Press, 1987.

[15] J. Schwarzer, V. Schulze, O. Vohringer, Finite element simulation of shot peening: A method to evaluate the influence of peening parameters on surface characteristics, in: ICSP-8, Garmisch-Partenkirchen, Germany, 2002, pp. 507-515.

[16] D. Lombardo, P. G. Bailey, The reality of shot peen coverage and dimple interactions, in: ICSP-6, San Francisco, USA, 1996, pp. 493-504. 